\title{
Innovation tut Not
}

\author{
Wolf Rainer Wendt
}

\begin{abstract}
Neuerungen bringen oft Unruhe in ein austariertes System. Dennoch garantieren nur Innovationen das Überleben letztlich auch der sozialen Unternehmen.
\end{abstract}

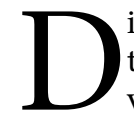
ie gegenwärtigen Sozialreformen, so wie sie politisch in die Wege geleitet werden, führen nicht von allein zu Neuerungen in den Organisationen der Sozialwirtschaft und in der von ihnen geleisteten humandienstlichen Versorgung. Neuerungen sind indes nötig, um einem sich ändernden Bedarf im Wandel der Lebensverhältnisse nachzukommen.

Innovationen bezeichnen neue Produkte oder Verfahren, die sich in Konkurrenz zu vorhandenen Produkten oder Verfahren durchsetzen. Ein bloß zusätzliches Angebot stellt keine Innovation dar. Der Frage ist nachzugehen, was im sozialwirtschaftlichen Bereich an Innovationen erwartet werden kann, wo Bedarf an ihnen besteht und wie sie zustande kommen.

\section{Im Wettbewerb um Lösungen}

Im System der sozialen Versorgung unterscheiden wir zwischen »services in cash « und »services in kind «. Bei Erörterung sozialwirtschaftlicher Innovationen beziehen wir uns auf die sächlichen Einrichtungen und Dienste, die personenbezogen vorgehalten werden. Die Geldleistungen sind zwar der hauptsächliche Gegenstand der po-

\begin{tabular}{|l|} 
Prof. Dr. Wolf \\
Rainer Wendt ist \\
Vorsitzender der \\
Deutschen Gesell- \\
schaft für Sozialar- \\
beit . V. Und Ho- \\
norarprofessor der \\
Eberhard Karls Uni- \\
versität Tübingen. \\
Er ist Herausgeber \\
der Edition »For- \\
schung und Ent- \\
wicklung in der \\
Sozialwirtschaft" \\
sowie Autor zahl- \\
reicher Bücher und \\
Fachaufsätze zur Sozialen Arbeit und zur SOZIAL- \\
E-Mailwwendt@ngi-portabledsl.de
\end{tabular}

litischen Debatte, in der es um die Reform der sozialen Sicherungssysteme geht. Änderungen in der Art und Weise der finanziellen Absicherung und Unterstützung, etwa die Umstellung bei der Zahlung von Arbeitslosen- und Sozialhilfe oder die Einführung der Praxisgebühr im Gesundheitswesen, stellen aber keine Innovationen in dem Sinne dar, in dem der Begriff durch den österreichischen Nationalökonomen Joseph Schumpeter in Gebrauch genommen und seither verwendet wird. Innovationen werden nicht politisch ausgehandelt und eingeführt; sie sind das Ergebnis einer Umstellung in der Praxis, also in der Art und Weise, wie etwas »gemacht $«$ wird.

Schumpeter nannte die Einführung neuer Produkte und Verfahren in der Wirtschaft einen Vorgang »schöpferischer Zerstörung « bestehender Strukturen. Innovation ist demnach ein Prozess, in dem die Planung, die Erzeugung und ein »Durchsetzen neuer Kombinationen « erfolgt. Innovator ist der schöpferische Unternehmer. Er ist dem Wettbewerb ausgesetzt; der Wettbewerb sorgt für Innovation.

Das Neue setzt sich im Wettbewerb gegen das Alte durch. Der kreative Unternehmer erfindet oder greift eine Erfindung auf, führt eine neue Methode der Produktion ein, organisiert den Herstellungsprozess neu und macht ein neues Geschäftsfeld auf. Ein innovatives Produkt setzt sich am Markt durch, und das Unternehmen profitiert davon solange, bis auch die Konkurrenz entsprechende Produkte anbietet: Die Innovation hat sich verbreitet und verliert mit der Zeit ihren Vorzug. Zusammenfassend erklärte Schumpeter die Innovation als das »Aufstellen einer neuen Produktionsfunktion « (siehe zu diesem Verständnis Wendt 2005.)

Vom ökonomischen und technologischen Konstrukt »Innovation « ist der lockere Umgang mit dem Wort zu unterscheiden. Alle Welt will heutzutage innovativ sein. In der Fülle der Waren und Dienste, die angeboten werden, fällt nur auf, was mit dem Anschein des Neuen daherkommt. Im sozialen Bereich lässt sich jeder Vorschlag, etwas anders als bisher zu machen, mit dem Attribut »innovativ«schmücken. Wenn sich Veränderungen in der Lebenspraxis der Gesellschaft durchsetzen, lässt sich durchaus von sozialen Innovationen sprechen. So ist der Begriff der Sozialinnovation auch besetzt. Er bezeichnet »neue Wege, Ziele zu erreichen, insbesondere neue Organisationsformen, neue Regulierungen, neue Lebensstile, die die Richtung des sozialen Wandels verändern, Probleme besser lösen als frühere Praktiken« (Zapf 1989, 177). Der häufig erhobene Anspruch, sozial innovativ zu sein, bedeutet aber oft nur, kreativ zu sein und mit neuen Ideen aufzuwarten. Invention wird mit Innovation verwechselt; ein guter Einfall hat aber nicht ohne weiteres in der Realität Bestand. 
In der organisierten sozialen Versorgung geht es um die Technologie und die Produkte, mit denen der Versorgungsauftrag erfüllt wird. Innovationen sind hier tatsächlich das Bessere, welches das bisher mehr oder minder Gutes ersetzt und zu verdrängen vermag. Sozialwirtschaftlich wird mit Produkten und Verfahren ein gesellschaftlich ausgemachter Bedarf gedeckt. Dafür wird beständig nach angemessenen Lösungen gesucht. Wer sie bietet, konkurriert mit der seitherigen Bedarfsdeckung, die nicht ohne weiteres aufgegeben wird. Eine neue Art und Weise der Leistungserbringung muss sich durchsetzen - im Wettbewerb unter Anbietern, bei den nachfragenden Leistungsträgern und in der sozialpolitisch interessierten Öffentlichkeit, die sich nicht zuletzt als Anwalt der Nutzer geriert.

Ein Versorger im sozialen Feld bedient zugleich vielfältig normierte und sich ändernde Erwartungen. Wenn wir mit Schumpeter fragen: Wie wandlungs- und innovationsfähig sind die Strukturen der Wohlfahrtsindustrie? dürfen wir nicht übersehen, dass der Sozialmarkt, auf dem sie mit neuen Produkten und Verfahren auftreten kann, ein regulierter ist.

Die Nachfrage, der ein Dienstleister nachkommt, lässt sich nicht einfach durch das Angebot der gemeinnützigen oder auch privat-gewerblichen Akteure steuern. Sie werden im Rahmen von Versorgungsaufträgen bezahlt, auf welche sie nur auf dem Umweg über die Politik Einfluss nehmen können. Andererseits werden in der sozialpolitischen Debatte über drängende Probleme die praktischen Lösungen in der Art und Weise der Leistungserbringung nicht gefunden. Hier hat der Wettbewerb unter Anbietern seine Funktion. Eine sozialwirtschaftliche Innovation etwa in der Jugendhilfe, der Behinderten- oder der Altenhilfe wird dem allgemeinen Auftrag in qualitativ neuer Weise gerecht und setzt sich deshalb durch.

\section{Neuerung, von oben angestoßen}

Das besondere Verhältnis von Auftrag und Dienstleistungsangebot in der Sozialwirtschaft führt dazu, dass der Anstoß zu Innovationen häufig aus der Politik und vom Gesetzgeber erfolgt. Wer rechtsgemäß handeln und seine institutionalisierte Beteiligung an der Ausführung sozialpolitischer Maßnahmen sichern will, neigt zu einem »nachahmenden Isomorphismus«. Die Leistungserbringer im korporatistischen System der Wohlfahrtspflege bewegen sich erst, wenn sie »von oben « dazu genötigt werden.

Ein Beispiel: Die vom Gesetzgeber im Zuge der Gesundheitsreform geforderte Integrierte Versorgung gemäß § 140 SGB V führt zu neuen sektor- und fachübergreifenden Lösungen. Die Krankenkassen schließen Verträge ab, in denen sich die Vertragspartner »zu einer qualitätsgesicherten, wirksamen, ausreichenden, zweckmäßigen und wirtschaftlichen Versorgung der Versicherten verpflichten « (§ 140 b Abs. 3 SGB V). Die Ausgestaltung der Integrierten Versorgung in diesem Rahmen (vgl. zur Vertragsgestaltung Bohle 2005) ist dabei in einer vielseitigen Weise möglich. In der Folge gibt es Innovationen: Krankenhäuser organisieren sich neu zu einem Produkt genannt »Gesundheitszentrum «; es bilden sich Ärztenetze, Medizinische Versorgungszentren und andere Verbünde von Leistungserbringern.

Ein weiteres Beispiel: Der Gesetzgeber hat den Weg von einer einrichtungszentrierten zu einer personenzentrierten Finanzierung eröffnet und damit den Wechsel von Sachleistungen zu Geldleistungen in der Subjektförderung ermöglicht. Dabei lösen Budgets die herkömmliche Alimentation $\mathrm{ab}$, und mit ihnen können die berechtigten Nutzer innovativ bedient werden. Insoweit diese die Wahl haben, müssen sich die Leistungserbringer in Konkurrenz untereinander anstrengen, mit ihren Produkten bei den Kunden »anzukommen«. Bekanntlich ermöglicht § 17 SGB IX grundsätzlich ein (trägerübergreifendes) Persönliches Budget überall im Sozial- und Gesundheitswesen. Die Gestaltung von komplexen Angeboten, die sowohl den individuellen, subjektiven Bedürfnissen von Budgetnehmern als auch dem objektiven Zweck einer Rehabilitation nachkommen, ist offen, »neue Kombinationen « in der Erbringung von Leistungen der Krankenbehandlung, der medizinischen Rehabilitation, der Teilhabe am Arbeitsleben, der Teilhabe am Leben in der Gemeinschaft, bei Pflegebedürftigkeit können sich durchsetzen. Allerdings gibt es eine Menge Hindernissen bei der Einführung der Budgets und auf sie bezogener Innovationen (vgl. Büscher u. a. 2005).

Ein anderes Feld der Betätigung, das politisch favorisiert wird, ist die Frühförderung von Kindern. Sie kann therapeutisch und pädagogisch kindzentriert betrieben werden. Aber auch in Konkurrenz zu einem solchen Vorgehen familienzentriert aufgrund der Erfahrung, dass mangelnde Befähigung von Eltern ein hauptsächliches Risiko für Kinder darstellt. Genannt sei hier beispielsweise STEEP als Frühinterventionsprogramm zur Unterstützung

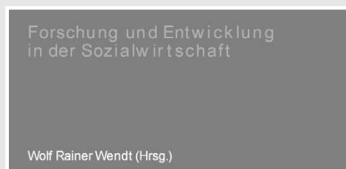

Innovation in der sozialen Praxis

$\mathbf{D}^{\text {Nomos }}$
Wolf Rainer Wendt (Hg.): Innovation in der sozialen Praxis. Bedingungen und Möglichkeiten der Erneuerung in Kontexten Sozialer Arbeit. Nomos Verlagsgesellschaft, Baden-Baden 2005, 145 Seiten. 27,- Euro. ISBN 3-8329-1514-1.

Innovationen sind die Stärke der produzierenden Wirtschaft. Sie stehen für Zukunftsfähigkeit. Innovative Lösungen werden aber auch gebraucht, um die soziale Versorgung für die Zukunft weiterzuentwickeln und zu sichern. Welche Bedeutung Innovationen im Wandel sozialer Verhältnisse und bei der Neustrukturierung sozialberuflicher Arbeit und sozialwirtschaftlicher Leistungserbringung zukommt, beleuchten Beiträge eines neuen Bandes aus der Reihe »Forschung und Entwicklung in der Sozialwirtschaft «. In einem innovationstheoretischen Teil des Herausgebers Wolf Rainer Wendt wird das Verständnis von Innovation in Wirtschaft, Technik und Gesellschaft erörtert. Innovationsprozesse in einzelnen Gebieten Sozialer Arbeit und den Ebenen der organisierten sozialen Versorgung sind Gegenstand von Beiträgen aus der Praxis. Fazit der Artikel: Soziale Arbeit ist zukunftsfähig, wenn sie mit neuen Lösungen dem Wandel ökonomischer und gesellschaftlicher Verhältnisse begegnet. 
der elterlichen Kompetenz von »Risikofamilien«, das gegenwärtig als Forschungsprojekt von zwei Fachhochschulen in Kooperation mit Beratungsstellen umgesetzt und vom Bundesministerium für Bildung und Forschung gefördert wird. Das Programm (»Steps towards effective and enjoyable parenting «) stammt aus den USA und ist von Martha Erickson an der University of Minnesota entwickelt worden. Frühförderung ist im Sozial- wie im Bildungswesen geboten und gewollt, also können passende Produkte als Verfahrensinnovationen zum Zuge kommen.

Für sie wird eine unabhängige Forschungs- und Entwicklungsarbeit gebraucht. Denn es besteht die Gefahr kurzlebiger Anpassungsinnovationen, wenn auf eine gerade vorherrschende politische Linie und die daraus resultierende Bereitstellung von Mitteln rasch mit einem darauf zugeschnittenen Produkt geantwortet wird. Etwa wenn in der Öffentlichkeit geschlossene Heime für schwererziehbare Kinder gefordert werden oder wenn auf die Not der Arbeitslosigkeit mit Qualifizierungsangeboten geantwortet wird, die finanziell gefördert werden, aber die Betroffenen nicht wirklich weiterbringen. Bei legislativen Vorgaben und gegenüber politischen Reformvorhaben ist sozialunternehmerische Bewegungsfreiheit in der Entwicklung von Innovationen angebracht.

In einem Vergleich von Einsätzen von Non-Profit-Organisationen in der lokalen Beschäftigungspolitik in Los Angeles und in Berlin stellte eine Forschergruppe sarkastisch fest, dass sich die Organisationen leicht zu bloßen Vollstreckern der Politik machen lassen, allerdings in unterschiedlicher Weise: Die engagierten Organisationen in Los Angeles könnten "mehrheitlich als pragmatische Trüffelschweine charakterisiert werden, die auf der Suche nach lukrativen Aufträgen und zur Sicherstellung ihrer Mission dazu bereit sind, aktiv das beschäftigungspolitische Feld zu durchwühlen und umzugraben, um sich neue Handlungsfelder zu erschließen«. In Berlin dagegen könne man von "staatsnahen Minenhunden « sprechen, »die sich gehorsam in die von den Verwaltungen angelegten beschäftigungspolitischen Minenfelder zwängen lassen, um von dort sodann entweder Vollzug zu melden und die brauchbaren Programme zu apportieren - oder an der kurzen Leine des Zusätzlichkeitskriteriums den Beweis zu erbringen, dass auch dieses beschäftigungspolitische Projekt zur Beseitigung der Massenarbeitslosigkeit ein Rohrkrepierer war« (Eick u. a. 2004, 179).

\section{Neuerung, von unten eingeführt}

Der größte Teil sozialer Versorgung wird informell geleistet. Dabei finden sich immer wieder neue Lösungen, die nur deshalb unbemerkt bleiben, weil sie das formelle System nicht berühren. Es kann aber von sich aus auf die Ansätze zugreifen, die es jenseits seiner Organisation gibt. Insbesondere die Vielfalt des Bürgerengagements generiert Innovationen. Bürgerschaftliches Engagement stellt politisch wie sozialwirtschaftlich ein $»$ Lernprojekt des Sozialstaates « dar (Hummel 1995, 14 ff.). Wie in der Erwerbswirtschaft die selbstständige Existenzgründung gefördert wird, sollte auch im sozialen Bereich individuelle und gemeinschaftliche Kreativität in der Selbsthilfe forciert und Eigeninitiative belohnt werden.

»Von unten« sind in den vergangenen Jahren verschiedene neue Wohnformen als Wohn- und Hausgemeinschaften von Menschen entstanden, die im Alter oder bei Behinderung oder bei drohender oder eingetretener Obdachlosigkeit selber nach für sie passenden Lösungen suchten. Dabei mussten eine Menge Hindernisse überwunden werden; nicht zuletzt standen und stehen dem Gemeinschaftswohnen Vorschriften im Wege, die für Heime gelten. Man findet sich zwar informell zusammen, damit das Vorhaben aber Bestand hat, braucht man formale Regelungen, auch in Hinblick auf die Inanspruchnahme professioneller Dienstleistungen. Exemplarisch für eine Innovation von Bürgerseite sei eine Entwicklung in der Schweiz genannt (vgl. Abbildung Seite 22).

Neue Wohnformen für alte Menschen sind in den 1990er-Jahren von Seniorengenossenschaften in Baden- 
Manche Arbeitsbereiche können überhaupt nur mit neuen Strukturen aufrechterhalten werden. Nehmen wir den Wandel der offenen Kinder- und Jugendarbeit in den letzten Jahren (vgl. Düx/Rauschenbach/Züchner 2002). Die »Komm-Struktur « der Jugendhäuser herrscht nicht mehr vor; mit den informations- und kommunikationstechnologischen Möglichkeiten von heute lassen sich virtuelle Vernetzungen gestalten, in denen und mittels derer Treffen, Events und andere Veranstaltungen zustande kommen. In Baden-Württemberg sind regionale Jugendagenturen entstanden, die über ein Internetportal erreicht tungsangebote insbesondere in Frage des Übergangs von Schule in Beruf und Arbeitswelt bereitstellen. Die Jugendagentur wird als »Koordinierungsnetzwerk « verstanden und erfordert naturgemäß ein anderes Management als das eines Jugendhauses (vgl. Hintermair 2004). Dass es vergleichbare Neustrukturierungen in der Altenpflege gibt (Stichwort »virtuelles Altenheim»), sei nur angemerkt (Greuél/Blechschmidt 2003).

Manche verstreute spezialisierte Leistungsangebote lassen sich dadurch wirtschaftlich und bedarfsentsprechend besser weiterführen, indem sie gebündelt ihre Synergie entfalten können. Das neue Produkt nennt sich dann Kompetenzzentrum. Die Bezeichnung bezieht sich gleichermaßen auf die Befähigung, die professionell eingebracht wird, und auf die Befähigung, die gepflegt und angestrebt wird. Kompetenzzentren bilden die Mitte eines Netzwerks und sind ein Kommunikationsknoten, der das Wissen und Können von Experten einerseits und informell vorhandenen Rat andererseits zusammenführt. Das Zentrum ist (drittens) dazu da, Wissen und Können zu erzeugen, Rat zu akkumulieren. Die Ressourcen der Unterstützung werden beispielsweise in einem Kinder- und Familienhilfezentrum gebündelt (Langhanky u. a. 2004). Beratung in dem doppelten Sinn der Bereitstellung von Rat und des Sichberatens von Menschen bietet ein Familienkompetenzzentrum. Mit ihm verankert sich das fachliche Angebot in dem Erfahrungsfeld von Lebensgemeinschaften, Eltern und Kindern und stellt sich besser auf sie ein (siehe Wendt 2005 a). Statt funktionszentriert wird prozessorientiert gearbeitet. So auch in polyvalenten Sozialzentren, musterhaft in Zürich (vgl. Stocker 2000, Waldvogel 2004).

Wenn es um die Infrastruktur der sozialen Versorgung geht, lassen sich Neuerungen insbesondere auf kommunaler Ebene dadurch erreichen, dass die öffentliche Hand, werden und für Jugendliche Informations- und Bera-

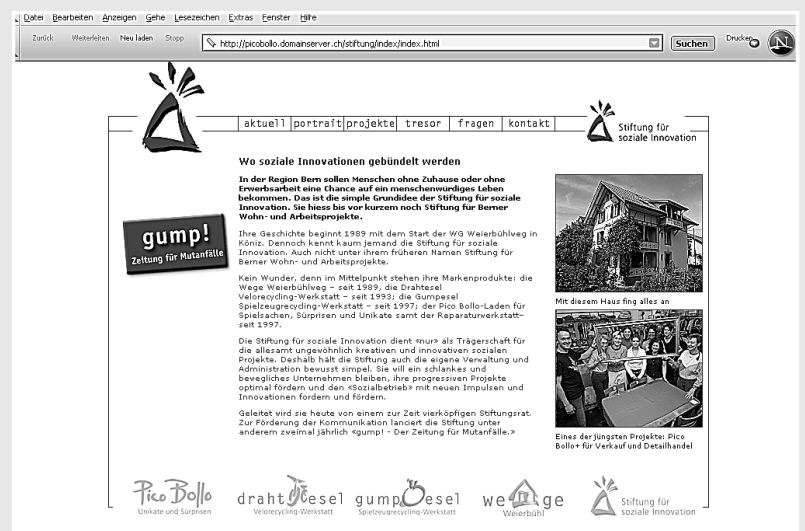

Bürgerschaftliches Engagement bringt immer wieder soziale Innovationen hervor. Beispiel Schweiz: Um eine Wohngemeinschaft für obdachlose Menschen bei Bern zu tragen, gründeten zwei Personen 1989 die Stiftung für Berner Obdachlose. Beschäftigungsangebote kamen hinzu, weshalb die Organisation seit 1998 Stiftung für Berner Wohn- und Arbeitsprojekte hieß. Daraus ist, nachdem weitere soziale Unternehmen angeschlossen wurden, im Juni 2005 die »Stiftung für soziale Innovation « geworden. In ihren Aktivitäten verbindet sie Wohnmöglichkeiten und Werkstätten mit Läden für den Verkauf von Produkten und mit Schulungsprogrammen für Erwerbslose (Internet http://picobollo.domainserver.ch/stiftung).

lichen Vorhaben und der Lebenswirklichkeit der Menschen leicht auftut.

\section{Projekt ist nicht gleich Innovation}

Im sozialen Bereich gelten Projekte zumeist als innovative Vorhaben. Mit einer gewissen Berechtigung wird, wer mit dem Entwurf einer Neugestaltung sozialer Aktivität hervortritt, mit Vorschusslorbeeren bedacht. Innovationsbedarf besteht schließlich, und neue Ansätze der Bedarfsdeckung sind daher stets willkommen.

Außerhalb der Sozialen Arbeit haben Projekte nicht die gleiche Bedeutung. Man spricht etwa bei einem Bauprojekt oder einem Verkehrsprojekt kaum je von einer Innovation. Ganz im Gegenteil werden solche Vorhaben im sozialen Umfeld oft als Beeinträchtigung von Lebensqualität

gemeinnützige Anbieter und Bürgerinitiativen zusammenwirken. Im lokalen Umfeld sind »human service innovations « auch eine Sache der Sozialplanung, der sozialraumorientierten Arbeitsteilung und von Public-PrivatePartnership. Dazu gibt es internationale Erfahrungen (Poole et al 2002, Richie/Alperin 2002) und gute Beispiele hierzulande (Leitner/Richter 2004, Hummel 2005). Produktentwicklung im Dialog mit den Bürgern hilft überdies die Kluft vermeiden, die sich zwischen amt- empfunden. Die Besserung, die mit der Realisierung solcher Projekte eintritt, kommt gewöhnlich nicht den unmittelbar Betroffenen zugute. Projekte der Sozialen Arbeit hingegen beginnen bei der Lage einer Bevölkerungsgruppe oder des lokalen Gemeinwesens und sollen eine neue Qualität für das Leben der Betroffenen bringen.

Solange diese neue Qualität nicht tatsächlich erreicht ist und sich in der Realität behauptet, liegt die Innovation 
noch nicht vor. Im Projektstadium befindet sie sich in einer Laborsituation. Es wird experimentiert und ist bestenfalls in der Erprobungsphase, in der viele reale Gegebenheiten noch ausgeklammert sind: Man hat eine geeignete Personengruppe ausgesucht, die Problematik selektiert, einen passenden Ort gefunden und die Beteiligten sind besonders motiviert. Es muss erst noch bewiesen werden, dass der Erfolg des Projekts auch im Normalfall des Alltags und rauer sozialer Wirklichkeit eintritt.

Indes braucht man für nachhaltige Innovationen in der sozialen Versorgung sehr wohl den Versuch unter Laborbedingungen und das Modellvorhaben als eine beispielhafte Erprobung im Feld. Allenthalben fördern denn auch Bund und Land sowie private Stiftungen modellhafte Projekte, und die Europäische Union legt für Beschäftigungsund Bildungsvorhaben, für die Jugend, für Integration und gegen soziale Exklusion entsprechende Programme auf.

Dem Erprobungscharakter gemäß erfolgt die Förderung von Projekten zeitlich begrenzt. Es liegt in der Natur von Modellversuchen, dass sie nicht unbefristet finanziert werden. Man muss sich also rechtzeitig um eine Übersetzung in die Regelversorgung kümmern, für deren beabsichtigte Änderung ein Projekt eigentlich bezahlt wird. In der Sozialen Arbeit ist immer wiederkehrend die Klage zu hören, dass Vorhaben nicht weitergeführt werden können und eine Maßnahme, die sich bewährt hat, eingestellt wird. Gewöhnlich besteht der Erfolg eben nur in einer gut geleisteten Arbeit und nicht darin, dass sie im Sinne Schumpeters auf ihrem Gebiet die Konkurrenz aussticht.

Zum Charakter von Innovationen gehört, dass sie sich durchsetzen. Sie verdrängen gewohnte Produkte oder Verfahren, setzen sich an ihre Stelle. Wenn soziale Projekte eingeführt und »bewilligt« werden, haben sie aber häufig gar nicht den Anspruch, an die Stelle einer vorhandenen Praxis zu treten. Sie sollen nicht mit ihr wetteifern, sondern sie eher ergänzen. Der Anschein von Konkurrenz wird oft vermieden, weil im korporatistischen Interessengeflecht der bedrohliche Anspruch die Chance der Förderung eines Projekts mindern würde. Dann ist es kein Wunder, wenn am Ende die übliche Praxis bleibt, wie sie ist.

Wenn man schon in Diensten für Menschen den offenen Wettstreit meidet, sollte man wenigstens in und mit gesellschaftlichen Anstrengungen konkurrieren, den drängenden Problemen mit neuen Lösungen zu begegnen. Der Anspruch sozialwirtschaftlicher Neuerungen richtet sich letztlich auf die Qualifizierung organisierter Daseinsvorsorge und sozialer Entwicklung.

Es besteht ein wechselseitiger Zusammenhang von sozialwirtschaftlichen Neuerungen in der Gestaltung von Dienstleistungen und sozialen Innovationen im Leben und Handeln von Menschen. Wenn sie sich in neuer Weise engagieren und ihr Einsatz in kooperativer Vernetzung in Angebotsstrukturen eingebunden wird, lassen sich damit neue Produkte in der Versorgung erstellen. Und umgekehrt fördert und unterhält eine professionell betriebene Vernetzungsarbeit das partikulare Engagement vieler Bürgerinnen und Bürger in gemeinsamen wie auch in eigenen sozialen Belangen.

\section{Innovative Wohlfahrtsproduktion}

In der Sozialbranche geht es bei neuartigen Produkten, Arrangements und Verfahren gewissermaßen um die Technologie der Wohlfahrtsproduktion. Wohlfahrtsproduktion verstanden als Transformationsprozess, in dem aus den Ressourcen, die man beizieht, ein Ertrag an Lebensqualität und humaner Entwicklung erstellt wird.

\section{Literatur}

Bohle, Thomas: Vertragsgestaltung in der Integrierten Versorgung. Ecomed-Verlag, Heidelberg 2005.

Büscher, Andreas/Budroni, Helmut/Hartenstein, Almut/Holle, Bernhard: Probleme bei der Umsetzung von Innovationen im Pflegemarkt - sichtbar am Beispiel personenbezogener Budgets. In: bpa magazin, 11, 1, 2005. S. 12-13.

Düx, Wiebken/Rauschenbach, Thomas/Züchner, Ivo (Red.): Innovation in der Kinder- und Jugendarbeit. Schriftenreihe Jugendhilfe in NRW, Heft 1. Votum, Münster 2002.

Eick, Volker/Grell, Britta/Mayer, Margit/Sambale, Jens: Nonprofit-Organisationen und die Transformation lokaler Beschäftigungspolitik. Westfälisches Dampfboot, Münster 2004.

Greuèl, Marius/Ulrike Blechschmidt: Integriertes Versorgungsmanagement für ältere und chronisch kranke Patienten am Beispiel der netzinternen Koordinierungsstelle HomeCare Nürnberg im Praxisnetz Nürnberg Nord. Bundesmodellprojekt »Virtuelles Altenheim «. Eigendruck, Nürnberg 2003.

Hintermair, Christa: Netzwerk ohne doppelten Boden. In: ProjektArbeit, 3, 1, 2004. S. 33-38.

Hummel, Konrad: Das bürgerschaftliche Engagement als Lernprojekt des Sozialstaates. In: Hummel, Konrad (Hg.): Bürgerengagement. Lambertus, Freiburg 1995. S. 14-41.

Hummel, Konrad: Kommunale Sozialpolitik - was ist machbar? Thesen zur Innovationsfähigkeit der kommunalen Sozialpolitik am Beispiel Augsburg. In: Wendt, Wolf Rainer (Hg.): Innovation in der sozialen Praxis. Nomos, Baden-Baden 2005. S. 50-59.

Langhanky, Michael/Frieß, Cornelia/Hußmann, Marcus/ Kunstreich, Timm: Erfolgreich sozial-räumlich handeln. Die Evaluation der Hamburger Kinder- und Familienhilfezentren. Kleine-Verlag, Bielefeld 2004.

Leitner, Hans/Richter, Hanka: Eine Stadt bewegt sich! Über die Entwicklung integrierter, flexibler und regionalisierter Hilfen zur Erziehung in Frankfurt (Oder). Frankfurt (Oder) 2004.

Otto, Ulrich: Seniorengenossenschaften. Modell für eine neue Wohlfahrtspolitik? Leske und Budrich, Opladen 1995.

Poole, Dennis L. et al.: The Capacity of Community-Based Organizations to Lead Local Innovations in Welfare Reform: Early Findings from Texas. In: Nonprofit Management and Leadership, 12, 3, 2002. S. 261-276.

Richie, Nicholas D./Alperin, Diane E. (eds.): Innovation and Change in the Human Services. Second Edition. Charles C. Thomas Publ., Springfield, Ill. 2002.

Stocker, Monika: Plädoyer für eine zukunftsfähige Sozialpolitik im Jahrzehnt danach. Zürich 2000.

Waldvogel, Rosann: Von der Funktion zum Prozess. Zürichs umfassende Reform der öffentlichen Sozial- und Jugendhilfe. In: Blätter der Wohlfahrtspflege, 151, 3, 2004. S. 104-107.

Wendt, Wolf Rainer: Dimensionen sozialer Innovation. In: Innovation in der sozialen Praxis. Nomos, Baden-Baden 2005. S. 13-49.

Wendt, Wolf Rainer: Was Familienberatung leistet. Ihre Ökonomie und ihr Beitrag zur Kompetenz von Familien. 2005 a, als pdf-Datei online unter http://www.dgsinfo.de/pdf/wendt_familienberatung.pdf.

Zapf, Wolfgang: Über soziale Innovation. In: Soziale Welt, 40 , 1-2, 1989. S. 170-183. 
In der Bekämpfung von Armut weiß man, dass zwischen Einkommensarmut, Verhaltens- und Bildungsarmut zu unterscheiden ist und dass nachhaltige Lösungen nicht von Einkommenstransfers zu erwarten sind. Familien, die seit Generationen von Sozialhilfe leben, wird nicht mit mehr Geld aus ihrer Lage geholfen. Hier sind im Verfahren »neue Kombinationen« im Sinne von Schumpeter gefragt. Einzelne Maßnahmen gibt es genug, sozialpädagogisch oder sozialtherapeutisch Verhaltensprobleme zu lösen und den Mangel an Bildung zu beheben. Einen Schritt weiter gehen Programme, die sich bei und nach einer Krisenintervention des problematischen Familienlebens insgesamt annehmen. Innovativ darüber hinaus kann ein Vorgehen sein, das in Anerkennung der je individuellen Weise des Zurechtkommens von Personen oder Familien ihre Bereitschaft und Anstrengung herausfordert, selber mit professioneller Unterstützung eigene Wohlfahrt zu produzieren.
Die Herstellungsarbeit konzentriert sich dann auf den Haushalt und die Lebenswelt der Adressaten, und es ist ihr Produkt, das zustande kommt.

In den vergangenen Jahrzehnten ist die humandienstliche Versorgung im Prozess der Deinstitutionalisierung den Weg von stationären Einrichtungen zu ambulanten Dienstleistungen gegangen. Weitere Schritte führen zu neuen Kombinationen mit der Selbsthilfe und Eigenaktivität von Menschen in persönlicher und gemeinschaftlicher Lebensführung und Daseinsgestaltung. Hier besteht Innovationspotenzial.

In der Sozialwirtschaft, so können wir schließen, ist die Forschung und Entwicklung darauf anzulegen, Formen zu finden, in denen die Koproduktion von individueller Wohlfahrt bei vielfältig gegebenem Bedarf gelingen kann.

\section{Zukunftsperspektiven für den Dritten Sektor}

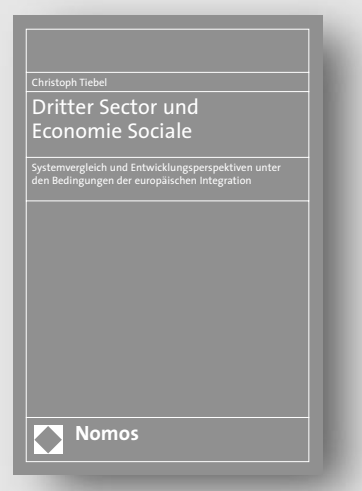

\section{Dritter Sector und Economie Sociale}

Systemvergleich und Entwicklungsperspektiven unter den Bedingungen der europäischen Integration

Von Prof. Dr. Christoph Tiebel, Leiter der Forschungsgruppe Gesundheits- und Sozialmanagement, FH Heilbronn und Unternehmensberater

2005, 167 S., brosch., 38,-€, ISBN 3-8329-1515-X

Trotz der gestiegenen Erwartungen an den Dritten Sektor von staatlicher und gesellschaftlicher Seite ließ sich eine exakte Einbindung des Sektors in das wirtschaftliche Gefüge der Gesellschaft bisher nicht vornehmen.

Das Buch ermöglicht einen Überblick über die verschiedenen Ansätze, mit denen sich der Dritte Sektor in Deutschland bewerten lässt und welche Entwicklungsper- spektiven das bestehende System durch die Prinzipien der Economie Sociale hat. Es befasst sich mit den systembedingten Besonderheiten des Dritten Sektors, die sich u.a. aus der Europäisierung ergeben. Es erfolgt eine Bewertung des Dritten Sektors mit Hilfe der Ansätze der Neuen Politischen Ökonomie. Zudem wird die Frage behandelt, ob das Prinzip der Economie Sociale für den Dritten Sektor zum Aufbau einer volkswirtschaftlich effizienten Bürgergesellschaft einen wichtigen Beitrag leisten kann.

Der Autor, Prof. Dr. Tiebel, beschäftigt sich als Leiter der Forschungsgruppe Gesundheits- und Sozialmanagement an der FH Heilbronn seit Jahren mit den spezifischen Gegebenheiten des Dritten Sektors und arbeitet als Unternehmensberater vornehmlich mit Non-Profit-Unternehmen aus dem sozialen Bereich zusammen. 